\title{
LINEAR CONVOLUTION INTEGRAL EQUATIONS WITH ASYMPTOTICALLY ALMOST PERIODIC SOLUTIONS
}

\author{
G. S. JORDAN, W. R. MADYCH AND R. L. WHEELER ${ }^{1}$
}

\begin{abstract}
ABSTRACr. Let $\mu$ be a bounded Borel measure and $f$ be asymptotically almost periodic. Conditions are found which ensure that certain bounded solutions of the linear convolution integral equation $g * \mu=f$ are asymptotically almost periodic. This result is also extended to the case where the measure $\mu$ is replaced by a tempered distribution $\tau$ for which convolution with bounded functions makes sense.
\end{abstract}

1. Classical convolution equations. Recently Fink and Madych [7] studied the asymptotic behavior as $t \rightarrow \infty$ of certain bounded solutions of the linear integral equation

$$
g * \mu(t) \equiv \int_{-\infty}^{\infty} g(t-s) d \mu(s)=f(t), \quad-\infty<t<\infty
$$

where $\mu$ is a bounded Borel measure on $R=(-\infty, \infty)$, and $f(t)$ belongs to $L^{\infty}=L^{\infty}(R)$ with $f(t) \rightarrow 0$ as $t \rightarrow \infty$. In this paper we show that the main result of [7, Theorem 3] may be extended in two directions. First, in this section (see Theorem 1) we show that Theorem 3 of [7] holds for more general forcing functions $f$ than are considered there. Then, in $\$ 2$ we show that the conclusion of Theorem 1 holds if the measure $\mu$ is replaced by a tempered distribution $\tau$ for which convolution with bounded functions makes sense.

We will use the notation of [7]. In particular, a function $g$ in $L^{\infty}$ is said to satisfy the tauberian condition $T$ if

$$
\lim _{t \rightarrow \infty, s \rightarrow 0}|g(t+s)-g(t)|=0 .
$$

If $m$ is a positive integer, we say that $f \in L^{\infty}$ satisfies property $M(f, m)$ if $\lim _{t \rightarrow \infty} f(t)=0$ and $\int_{0}^{\infty} t^{m-1}|f(t)| d t<\infty$. By $M(f, \infty)$ we mean that $M(f, m)$ holds for each positive integer $m$. If $f$ is an almost periodic function ( $f \in$ a.p.), $\exp f$ denotes the set of exponents of $f$ (see [6, Chapter 3]).

Also, $\mathfrak{N}$ denotes the space of bounded Borel measures. If $\mu \in \mathfrak{R}$, let $\hat{\mu}(\xi)=$ $\int_{-\infty}^{\infty} e^{-i \xi t} d \mu(t)$ be the Fourier transform of $\mu$ and $\Lambda(\mu)=\{\xi \in R: \hat{\mu}(\xi)=0\}$ be the set of zeros of $\hat{\mu}$. Finally, we say that $\mu \in \mathfrak{N}$ satisfies property $M \Lambda(\mu)$ if for every $\xi_{j} \in \Lambda(\mu)$ there exists a positive integer $m_{j}$ (necessarily unique) such that the function $\hat{\nu}_{j}$ defined by $\hat{\nu}_{j}(\xi)=\left(\xi-\xi_{j}\right)^{-m_{j}} \hat{\mu}(\xi)$ is the Fourier transform of a measure

Received by the editors March 6, 1979.

AMS (MOS) subject classifications (1970). Primary 45M05; Secondary 42A84.

Key words and phrases. Convolution integral equation, asymptotically almost periodic, tempered distribution.

${ }^{1}$ Partially supported by NSF Grant MCS 78-01330. 
$\nu_{j} \in \mathfrak{R}$ with $\hat{\nu}_{j}\left(\xi_{j}\right) \neq 0$. Note that if $\Lambda(\mu)=\varnothing$, then $\mu$ trivially has property $M \Lambda(\mu)$.

THEOREM 1. Suppose $g$ is a bounded solution of equation (1), where

(i) $\Lambda(\mu)$ has no finite accumulation point,

(ii) $\mu$ satisfies property $M \Lambda(\mu)$,

(iii) $f=f_{1}+f_{2}$ where $f_{1} \in$ a.p. and $f_{2}$ satisfies property $M(f, m)$ for $m=\sup \left\{m_{j}\right\}$ where the numbers $m_{j}$ are defined in the previous paragraph,

(iv) $g$ satisfies condition $T$.

Then $g=g_{1}+g_{2}$ where $g_{1} \in$ a.p. with $\exp g_{1} \subseteq \Lambda(\mu) \cup \exp f_{1}$, and $\lim _{t \rightarrow \infty} g_{2}(t)=$ 0 .

In the case where $f_{1}(t) \equiv 0$, Theorem 1 is Theorem 3 of [7]. (More restricted versions of [7, Theorem 3] where, in particular, $\Lambda(\mu)$ is finite are obtained in Jordan and Wheeler [8, Theorem 1], and in Levin and Shea's three part paper [9, Theorem 5c].) On the other hand, in the case where $f=f_{1}$ is a.p., a related result which guarantees that bounded, uniformly continuous solutions of (1) (with $\mu$ absolutely continuous) are a.p. was obtained by Lewitan [10]. (See, also, Doss [4] and Lemma 2 below.) Finally, the nature of bounded, uniformly continuous solutions of the homogeneous form of equation (1), i.e., with $f(t) \equiv 0$, was investigated by Beurling in [2], [1], and now constitutes one aspect of spectral analysis; see $[9, \S 8]$ for a brief discussion which includes references to appropriate literature.

Our proof of Theorem 1 consists of reducing the problem to considering separately the case where $f(t)=f_{2}(t) \rightarrow 0$ as $t \rightarrow \infty$, and the case where $f=f_{1}$ is a.p.

LEMMA 1. Suppose $g$ is a bounded solution of equation (1) which satisfies the tauberian condition $T$, and $f=f_{1}+f_{2}$ where $f_{1} \in$ a.p. and $\lim _{t \rightarrow \infty} f_{2}(t)=0$. Then there exists a function $h$ which is bounded, uniformly continuous on $R$, and which satisfies $h * \mu(t)=f_{1}(t)$ for $t \in R$.

The idea of Lemma 1 is implicitly contained in the discussion given in $\$ 20$, pp. 567-568 of [9]. For completeness we include the proof.

Proof. The $\varepsilon$-translation set of $f_{1}$ is

$$
T\left(f_{1}, \varepsilon\right)=\left\{s \in R:\left|f_{1}(t+s)-f_{1}(t)\right|<\varepsilon \text { for all } t \in R\right\} .
$$

Since $f_{1} \in$ a.p., we can find $s_{j} \in T\left(f_{1}, 1 / j\right)$ for $j=1,2, \ldots$, so that $\lim _{j \rightarrow \infty} s_{j}=$ $\infty$. Since $g$ is bounded and satisfies $(T)$, Lemma 3.2 of [9] guarantees that there exist a subsequence $\left\{s_{j_{k}}\right\}$ of $\left\{s_{j}\right\}$ and a bounded, uniformly continuous function $h$ on $R$ such that

$$
\lim _{k \rightarrow \infty}\left\{\sup _{|t|<d}\left|g\left(t+s_{j_{k}}\right)-h(t)\right|\right\}=0 \quad \text { for every } d>0 .
$$

Fix $t \in R$ and observe that by (1)

$$
g * \mu\left(t+s_{j_{k}}\right)=f_{1}\left(t+s_{j_{k}}\right)+f_{2}\left(t+s_{j_{k}}\right)
$$


for $k=1,2, \ldots$ Thus, if we let $k \rightarrow \infty$ in (3) and use $\mu \in \mathfrak{T},(2), s_{j_{k}} \in$ $T\left(f_{1}, 1 / j_{k}\right)$ and $f_{2}(t) \rightarrow 0$ as $t \rightarrow \infty$, we get that $h * \mu(t)=f_{1}(t)$. This completes the proof of Lemma 1 .

The following lemma relates properties of bounded, uniformly continuous solutions of equation (1) with $f \in$ a.p. to those of bounded, uniformly continuous solutions of the homogeneous equation

$$
g * \mu(t)=0, \quad t \in R .
$$

LEMma 2. Assume that $\mu \in \mathfrak{T}$. If every bounded, uniformly continuous solution $g$ of the homogeneous equation (4) is a.p., then, for each $f \in$ a.p., every bounded, uniformly continuous solution $g$ of equation (1) is a.p.

When $\mu(t) \equiv \int_{-\infty}^{t} k(s) d s$ with $k \in L^{1}(R)$, Lemma 2 is due to Doss [4, Lemma 2]. The proof for general $\mu \in \mathfrak{N}$ follows in exactly the same manner as is indicated by Doss in [4] for the case where $\mu$ is absolutely continuous. Namely, use Bochner's proof of Theorem 4 in [3] with the differential operator $\Lambda g$ replaced by the integral operator $K g=g * \mu$ throughout.

Proof of Theorem 1. By Lemma 1 there exists a bounded, uniformly continuous solution $h$ of $h * \mu=f_{1}$ on $R$. Since $\Lambda(\mu)$ has no finite accumulation point, every bounded, uniformly continuous solution $h_{1}$ of $h_{1} * \mu=0$ is a.p. (See, e.g., [9, Proposition 8.1].) Thus, by Lemma 2, $h \in$ a.p. It is easy to verify that $\exp h \subseteq$ $\Lambda(\mu) \cup \exp f_{1}$.

Now, define $G(t) \equiv g(t)-h(t), t \in R$. Then $G$ satisfies $G * \mu=f_{2}$ on $R$, and by Theorem 3 of [7], $G=G_{1}+G_{2}$, where $G_{1} \in$ a.p. with $\exp G_{1} \subseteq \Lambda(\mu)$ and $\lim _{t \rightarrow \infty} G_{2}(t)=0$. Thus, $g=g_{1}+g_{2}$ where $g_{1} \equiv G_{1}-h$ and $g_{2} \equiv G_{2}$ satisfy the conclusion of the theorem.

2. Extension to distributions. In this section we show that Theorem 1 holds if the measure $\mu$ is replaced by a tempered distribution $\tau$ for which, roughly, $g * \tau$ makes sense for bounded $g$.

We say that a tempered distribution $\tau$ on $R$ satisfies property $H$ if the convolution $\tau * \phi \in L^{1}(R)$ for every $\phi$ in the Schwartz space $\mathcal{S}=\mathcal{S}(R)$. (The definitions of $\mathcal{S}$, tempered distributions, the convolution $\tau * \phi$, and Fourier transforms of tempered distributions are all standard; e.g., see [5].) If $g \in L^{\infty}$, define the linear form $g * \tau: \mathcal{S} \rightarrow$ C by the formula $g * \tau(\phi)=g *(\tau * \tilde{\phi})(0)$ where $\tilde{\phi}(t) \equiv \phi(-t)$ and the first convolution on the right-hand side is taken in the classical sense.

LeMmA 3. If $\tau$ satisfies property $H$ and $g \in L^{\infty}$, then $g * \tau$ is a tempered distribution.

Proof. Since $|g * \tau(\phi)| \leqslant\|g\|_{\infty}\|\tau * \phi\|_{1}$, the lemma is true if the mapping $\phi \rightarrow \tau * \phi$ is continuous from $\delta$ into $L^{1}$.

Let $\psi$ be a function in $\mathcal{S}$ whose Fourier transform, $\hat{\psi}$, has the following properties: $\hat{\psi}$ is nonnegative, $\hat{\psi}(\xi)=1$ if $|\xi| \leqslant 1$, and $\hat{\psi}(\xi)=0$ if $|\xi| \geqslant 2$. For any positive number $r$ define $\psi_{r}$ by the formula $\psi_{r}(t)=r \psi(r t)$. Now suppose that $\left\{\phi_{n}\right\}$ is a sequence in $\mathcal{S}, \phi_{n} \rightarrow \phi$ in $\mathcal{S}$, and $\tau * \phi_{n} \rightarrow f$ in $L^{1}$. Clearly $\tau * \phi_{n} * \psi_{r}$ converges to 
$\tau * \phi * \psi_{r}=f * \psi_{r}$ in $L^{1}$ for each positive $r$. Now $\|\tau * \phi-f\|_{1}<\| \tau * \phi-$ $\tau * \phi * \psi_{r}\left\|_{1}+\right\| \tau * \phi * \psi_{r}-f \|_{1}$, and choosing $r$ sufficiently large it is clear that the right-hand side of the above inequality can be made arbitrarily small. Hence $\tau * \phi=f$. It follows that the mapping $\phi \rightarrow \tau * \phi$ has a closed graph in $\mathcal{S} \times L^{1}$ and hence must be continuous. (For the variant of the closed graph theorem used here see, for example, [5].)

Various properties of tempered distributions satisfying property $H$ should be clear from the definition. For example, the Fourier transform of such a distribution must be a continuous function.

Clearly, bounded measures and certain linear combinations of their derivatives are tempered distributions which satisfy property $H$. For example, consider the distribution whose Fourier transform $\hat{\tau}$ is given by

$$
\hat{\tau}(\xi)=\sum_{j=1}^{n} P_{j}(i \xi) \hat{\mu}_{j}(\xi)
$$

where the $\hat{\mu}_{j}$ 's are Fourier transforms of bounded measures $\mu_{j} \in \mathfrak{N}$, and each

$$
P_{j}(i \xi)=\sum_{l=0}^{m_{j}} a_{j, l}(i \xi)^{l}, \quad j=1, \ldots, n,
$$

is a polynomial in $(i \xi)$. Then

$$
g * \tau(t)=\sum_{j=1}^{n} \int_{-\infty}^{\infty} P_{j}(D) g(t-s) d \mu_{j}(s)
$$

where $P_{j}(D) g(t)=\sum_{l=0}^{m_{j}} a_{j, l} g^{(l)}(t)$ and $g^{(l)}(t)$ is the $l$ th derivative of $g$.

If $\tau$ enjoys property $H$, the definitions of $\Lambda(\tau)$ and property $M \Lambda(\tau)$ are analogous to those in $\S 1$ in the case where $\tau \in \mathfrak{N}$. Namely, $\Lambda(\tau)=\{\xi \in R: \hat{\tau}(\xi)=0\}$, and we say that $\tau$ satisfies property $M \Lambda(\tau)$ if for every $\xi_{j} \in \Lambda(\tau)$ there exists a positive integer $m_{j}$ such that the function $\hat{\nu}_{j}(\xi) \equiv\left(\xi-\xi_{j}\right)^{-m_{j}} \hat{\tau}(\xi)$ is the Fourier transform of a tempered distribution having property $H$ and $\hat{\nu}_{j}\left(\xi_{j}\right) \neq 0$.

THEOREM 2. Suppose $\tau$ satisfies property $H, g$ is a bounded solution of $g * \tau=f$ and the following are true:

(i) $\Lambda(\tau)$ has no finite accumulation point,

(ii) $\tau$ satisfies property $M \Lambda(\tau)$,

(iii) $f=f_{1}+f_{2}$ where $f_{1} \in$ a.p. and $f_{2}$ satisfies property $M(f, m)$ for $m=\sup \left\{m_{j}\right\}$ where the numbers $m_{j}$ are defined in the previous paragraph,

(iv) $g$ satisfies condition $T$.

Then $g=g_{1}+g_{2}$ where $g_{1} \in$ a.p. with $\exp g_{1} \subseteq \Lambda(\tau) \cup \exp f_{1}$, and $\lim _{t \rightarrow \infty} g_{2}(t)=$ 0 .

Proof. Let $\phi(x)=e^{-x^{2}}$. Clearly, $g *(\tau * \phi)=f * \phi+f_{2} * \phi$. It follows from $(\tau * \phi)^{\wedge}(\xi)=\hat{\tau}(\xi) \hat{\phi}(\xi)$ and $\Lambda(\phi)=\varnothing$, that $\Lambda(\tau * \phi)=\Lambda(\tau)$. Also, for each $\varepsilon>0$, $T\left(f_{1} * \phi, \varepsilon\right) \supseteq T\left(f_{1}, \varepsilon /\|\phi\|_{1}\right)$, so that $f_{1} * \phi \in$ a.p.; in addition, by Lemma 4.7 of [6] $\exp \left(f_{1} * \phi\right)=\exp f_{1}$. Finally, Lemma 3 of [7] implies that $f_{2} * \phi$ satisfies property $M\left(f_{2} * \phi, m\right)$. Thus, Theorem 2 follows from Theorem 1 with $\mu=\tau * \phi$. 
For an example, consider the integrodifferential equation

$$
\sum_{l=0}^{n} \int_{-\infty}^{\infty} g^{(l)}(t-s) d \mu_{l}(s)=f(t), \quad t \in R,
$$

where $\mu_{l} \in \mathfrak{N}, l=0, \ldots, n$. Clearly, this equation is a special case of $g * \tau=f$ where $\tau$ has property $H$ and $\hat{\tau}(\xi)=\sum_{l=0}^{n}(i \xi)^{\prime} \hat{\mu}_{l}(\xi)$. We say that $\xi_{0} \in R$ is a zero of $\hat{\tau}(\xi)$ of multiplicity $p$ if

$$
\int_{-\infty}^{\infty}|t|^{p}\left|d \mu_{l}(t)\right|<\infty \quad(l=0, \ldots, n)
$$

and if

$$
\left(d^{j} / d \xi^{j}\right) \hat{\tau}(\xi)=0 \quad\left(\xi=\xi_{0} ; j=0, \ldots, p-1\right)
$$

but

$$
\left(d^{p} / d \xi^{p}\right) \hat{\tau}(\xi) \neq 0 \quad\left(\xi=\xi_{0}\right)
$$

Assume that each zero of $\hat{\tau}(\xi)$ has finite multiplicity and let $m(\leqslant \infty)$ be the supremum of the multiplicities of the zeros of $\hat{\tau}(\xi)$. Then an argument similar to the proof of Lemma 5 of [7] shows that property $M \Lambda(\tau)$ holds. Thus, if $\Lambda(\tau)$ has no finite accumulation point, if $f$ is as in Theorem 2, and if $g(t)$ is a solution of (2.2) a.e. on $R$ with $g^{(l)} \in L^{\infty}(l=0, \ldots, n-1)$ and $g^{(n-1)}$ locally absolutely continuous, then $g$ has the form described in Theorem 2 . We remark that the special case of equation (2.2) with $\mu_{n}$ the point-mass measure concentrated at $t=0$, has been considered previously in [8, Theorem 5] (see also [9, Theorem 5a]) when $\Lambda(\tau)$ is finite.

ACKNowledgement. The authors thank the referee for his useful suggestions.

\section{REFERENCES}

1. A. Beurling, Un théorème sur les fonctions bornées et uniformément continues sur l'axe réel, Acta Math. 77 (1945), 127-136.

2. Sur une classe de fonctions presque-périodiques, C. R. Acad. Sci. Paris 255 (1947), 326-328.

3. S. Bochner, $A$ new approach to almost periodicity, Proc. Nat. Acad. Sci. U.S.A. 48 (1962), 2039-2043.

4. R. Doss, On the almost periodic solutions of a class of integro-differential-difference equations, Ann. of Math. (2) 81 (1965), 117-123.

5. R. E. Edwards, Functional analysis, Holt, Rinehart and Winston, New York, 1965.

6. A. M. Fink, Almost periodic differential equations, Lecture Notes in Math., vol. 377, SpringerVerlag, Berlin, 1974.

7. A. M. Fink and W. R. Madych, On certain bounded solutions of $g * \mu=f$, Proc. Amer. Math. Soc. 75 (1979), 235-242.

8. G. S. Jordan and R. L. Wheeler, Linear integral equations with asymptotically almost periodic solutions, J. Math. Anal. Appl. 52 (1975), 454-464.

9. J. J. Levin and D. F. Shea, On the asymptotic behavior of the bounded solutions of some integral equations, I, II, III, J. Math. Anal. Appl. 37 (1972), 42-82; 288-326; 537-575.

10. B. Lewitan, On an integral equation with an almost periodic solution, Bull. Amer. Math. Soc. 43 (1937), 677-679.

Department of Mathematics. University of Tennessee, Knoxville, Tennessee 37916

Department of Mathematics, Iowa State University, Ames, Iowa 50011

Department of Mathematics, University of Missouri, Columbia, Missouri 65211 